\title{
Bose-Hubbard physics in synthetic dimensions from interaction Trotterization
}

\author{
L. Barbiero $\odot,{ }^{1,2, *}$ L. Chomaz $\odot,{ }^{3}$ S. Nascimbene $\odot,{ }^{4}$ and N. Goldman ${ }^{1, \dagger}$ \\ ${ }^{1}$ Center for Nonlinear Phenomena and Complex Systems, Université Libre de Bruxelles, CP 231, Campus Plaine, B-1050 Brussels, Belgium \\ ${ }^{2}$ ICFO - Institut de Ciencies Fotoniques, The Barcelona Institute of Science and Technology, Avinguda Carl \\ Friedrich Gauss 3, 08860 Castelldefels (Barcelona), Spain \\ ${ }^{3}$ Institut für Experimentalphysik, Universität Innsbruck, Technikerstrasse 25, 6020 Innsbruck, Austria \\ ${ }^{4}$ Laboratoire Kastler Brossel, Collège de France, CNRS, ENS-PSL University, Sorbonne Université, \\ 11 Place Marcelin Berthelot, 75005 Paris, France
}

(Received 26 July 2019; accepted 10 November 2020; published 8 December 2020)

\begin{abstract}
Activating transitions between a set of atomic internal states has emerged as an elegant scheme by which lattice models can be designed in ultracold atomic gases. In this approach, the internal states can be viewed as fictitious lattice sites defined along a synthetic dimension, hence offering a powerful method by which the spatial dimensionality of the system can be extended. Interparticle collisions generically lead to infinite-range interactions along the synthetic dimensions, which a priori precludes the design of Bose-Hubbard-type models featuring on-site interactions. In this paper, we solve this obstacle by introducing a protocol that realizes strong and tunable "on-site" interactions along an atomic synthetic dimension. Our scheme is based on pulsing strong intraspin interactions in a fast and periodic manner, hence realizing the desired on-site interactions in a digital (Trotterized) manner. We explore the viability of this protocol by means of numerical calculations, which we perform on various examples that are relevant to ultracold-atom experiments. This general method, which could be applied to various atomic species by means of fast-response protocols based on Fano-Feshbach resonances, opens the route for exploration of strongly correlated matter in synthetic dimensions.
\end{abstract}

DOI: 10.1103/PhysRevResearch.2.043340

\section{INTRODUCTION}

Quantum simulation offers a method by which complex phenomena can be analyzed using well-designed quantum systems [1]. Various physical platforms have been envisaged in this vast program, including ultracold atoms in optical lattices, trapped ions, superconducting qubits, photonic devices, quantum dots, and point defects in diamond. Many of these versatile systems can be arranged in lattices of various geometries, allowing for particles to move in $D=1,2$, or 3 spatial dimensions; see, for instance, the reviews in Refs. [2,3]. Beyond these physical lattices, it was suggested that motion can also be activated along a "synthetic dimension" associated with internal degrees of freedom [4-6]. This ingenious feature substantially extends the quantum-simulation toolbox: Not only does it allow for the study of two-dimensional (2D) phenomena (such as the quantum Hall effect) starting from a 1D lattice system $[5,7,8]$, but also it offers the appealing possibility of exploring higher-dimensional $(D>3)$ physics in the laboratory [4,9-11].

In ultracold atoms, several schemes have been proposed to increase the effective dimensionality of the system. The

\footnotetext{
*luca.barbiero@icfo.eu

†ngoldman@ulb.ac.be
}

Published by the American Physical Society under the terms of the Creative Commons Attribution 4.0 International license. Further distribution of this work must maintain attribution to the author $(s)$ and the published article's title, journal citation, and DOI. original proposals of Refs. [4,5] suggested using internal atomic states ("spins") as fictitious lattice sites along a synthetic dimension, the motion along which can be activated by coupling these states with Raman transitions. This scheme was then generalized to long-lived electronic orbital states coupled through an optical clock transition [12,13], rotational states of ultracold molecules coupled by microwaves [14], discrete momentum states coupled by Bragg transitions [15], and harmonic-oscillator states coupled by shaking of the trap $[7,16]$. Experimental realizations of synthetic dimensions revealed diverse phenomena, including chiral edge currents [17-19], Anderson topological insulators [20], topological solitons [21], and a flux-dependent mobility edge in disordered chains [22]. In parallel, similar techniques were developed in photonics $[6,10,23-26]$ and very recently in the solid state [27].

Until now, most experiments exploiting atomic synthetic dimensions have been operating in the weakly interacting (or noninteracting) regime, where physical observables can be described in terms of a single-particle band structure. However, recent theoretical works have suggested that intriguing physical phenomena can arise in these settings upon taking strong interparticle interactions into account. In the case of fermionic species, synthetic dimensions with spin-independent interactions were shown to generate special crystalline states [28], exotic edge currents [29-31], orbital magnetism [32], and symmetry-protected topological insulating states [33]. In the case of bosonic species, where collisions in the same internal state are also allowed, theorists have predicted supersolid states [34], vortex-hole duality phenomena [35], and analog 
Haldane phases [36]. Experimental studies of interaction effects in synthetic dimensions have been recently reported in the contexts of momentum-state lattices $[37,38]$ and optical lattice clocks [39].

Importantly, two-body contact interactions generically translate into infinite-range interactions along synthetic dimensions spanned by atomic internal states [5]. In fact, for certain atomic species, the scattering length is independent of the atomic internal states involved in the collisions, hence leading to $\mathrm{SU}(M)$-invariant interactions (where $M$ refers to the number of internal states involved); see Refs. [40-42]. While infinite-range interactions lead to interesting phenomena, they effectively reduce the dimensionality of the synthetic dimension: Indeed, in the presence of strong interactions, spin-changing processes can be neglected, and the infinite-range nature of the interactions effectively reduces the synthetic dimension from 1D to 0D. Besides, the effects generated by $\mathrm{SU}(M)$ interactions are generically captured by mean-field approaches. These observations indicate that genuine $2 \mathrm{D}$ interacting quantum states, such as fractional quantum Hall $(\mathrm{FQH})$ states [43-45], cannot be created by combining a real lattice system (with on-site interactions) and a synthetic dimension with infinite-range interactions; the absence of $\mathrm{FQH}$ states in such a setting was analyzed numerically in Ref. [46] through the calculation of edge currents. Altogether, this indicates the importance of designing controllable on-site (or finite-range) interactions in synthetic dimensions [16,47], in view of exploring strongly interacting topological matter in these settings.

In this paper, we propose a realistic scheme realizing effective on-site interactions in a synthetic dimension spanned by atomic internal states. Our method builds on using multiple Fano-Feshbach resonances in atomic spin mixtures [48-50] to select and enhance intraspin interactions. Specifically, we propose to use this technology to pulse strong intraspin interactions in a fast and time-periodic manner (Fig. 1). As we demonstrate below, this Trotterization scheme effectively generates strong and constant on-site interactions along the synthetic (internal-state) dimension, hence realizing the Bose-Hubbard model in this simple setting. We validate this approach based on several examples that are relevant to ongoing experimental efforts, comparing the dynamics resulting from the full time-dependent Hamiltonian with that of the effective Hubbard-type model. In particular, we study the impact of the background infinite-range interactions, which will typically be present in experimental implementations. As illustrative examples, we consider the bosonic Mott-superfluid transition and the formation of an effective antiferromagnetic order in a single synthetic dimension, as well as the creation of a chiral Mott-Meissner phase in a synthetic ladder geometry with an effective magnetic flux. The latter setting, which features a combination of effective on-site interactions and artificial magnetic flux, constitutes a promising starting point for the study of $\mathrm{FQH}$ physics in synthetic dimensions.

\section{THE MODEL AND THE TROTTER PROTOCOL}

In this section, we introduce a general scheme by which onsite interactions can be generated in laser-induced synthetic (a)

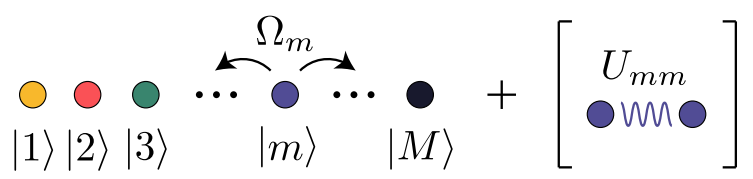

(b)

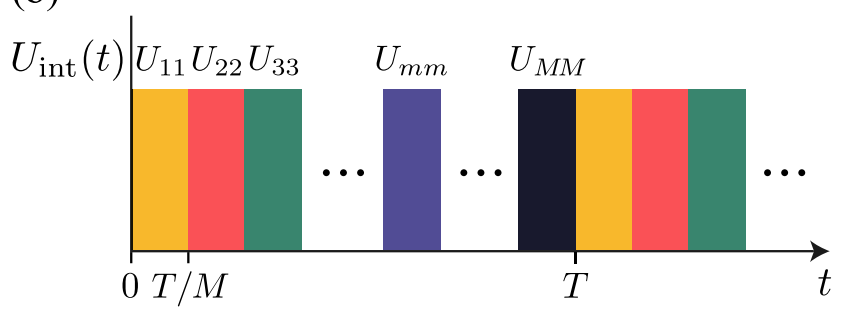

FIG. 1. (a) A synthetic dimension is spanned by $M$ internal states of an atom. "Hopping" matrix elements along the synthetic dimension, $\Omega_{m}$, are controlled by laser coupling, while intraspin interactions $U_{m m}$ are tuned by Fano-Feshbach resonances. The scheme assumes that interspin interactions $U_{m \neq n}$ (infinite-range interactions along the synthetic dimension) can be made small compared with all other energy scales. (b) The Trotterization scheme consists in pulsing intraspin interactions in a successive and time-periodic manner: In the first step, one activates $U_{11}$ only, then $U_{22}$ only, etc. The drive frequency $\omega=2 \pi / T$ is assumed to be the largest frequency in the system: $\hbar \omega \gg \Omega_{m}, U_{m m}$.

dimensions, hence realizing the Bose-Hubbard model for cold atoms in the absence of a physical lattice.

As illustrated in Fig. 1(a), we consider a bosonic atom with $M$ internal states, denoted $|m\rangle$ with $m=(1, \ldots, M)$. These (potentially many) internal states, or "spins," will be viewed as fictitious lattice sites aligned along a synthetic dimension [6]. The "hopping" along the synthetic dimension can be activated by coupling these internal states using a proper laser configuration, which then defines the corresponding hopping matrix elements $\Omega_{m}$. When considering an ensemble of such atoms, the system generically presents a wide variety of interaction processes, which typically depend on the internal states involved in the collisions; in the following, we neglect spin-changing collisions [51]. Considering a strongly confined atomic sample, such that its motional degrees of freedom are frozen in the ground state of the confining potential ("single-mode approximation"), we describe such a system by the extended Bose-Hubbard Hamiltonian

$$
\begin{aligned}
\hat{H}= & -\sum_{m=1}^{M}\left(\Omega_{m} \hat{b}_{m+1}^{\dagger} \hat{b}_{m}+\text { H.c. }\right) \\
& +\sum_{m} \frac{U_{m m}}{2} \hat{n}_{m}\left(\hat{n}_{m}-1\right)+\sum_{m \neq n} \frac{U_{m n}}{2} \hat{n}_{m} \hat{n}_{n},
\end{aligned}
$$

where $\hat{b}_{m}^{\dagger}\left(\hat{n}_{m}\right)$ is the creation (number) operator of a boson in the internal state $m$ and where the coefficients $U_{m n}$ characterize the various interspin $(m \neq n)$ and intraspin $(m=n)$ interaction strengths. Specifically, these coefficients are given by $U_{m n}=a_{m n}\left(4 \pi \hbar^{2} / \mathcal{M}\right) \int d^{3} \boldsymbol{x} w^{4}(\boldsymbol{x})$, where $a_{m n}$ is the scattering length associated with spin-conserving collisions in internal states $m$ and $n, \mathcal{M}$ is the mass of an atom, and $w(\boldsymbol{x})$ denotes the harmonic-oscillator ground-state wave function. In order for the single-mode approximation to be valid, we 
require the scattering lengths to satisfy $\left|a_{m n}\right| \ll l_{\mathrm{H}}$, where $l_{\mathrm{H}}$ denotes the characteristic length of the harmonic confinement. Importantly, these interaction coefficients are generically all of the same order in experiments, which leads to effectively infinite-range interactions along the synthetic dimension. This fundamental aspect precludes the physics of the conventional Bose-Hubbard model in laser-induced synthetic dimensions. While interactions can be tuned externally, using Feshbach resonances, the latter cannot directly produce the desired configuration, namely, strong and uniform intraspin interactions $U_{m m} \approx U \gg U_{m \neq n}$ for all states $m$.

The aim of our scheme is to artificially generate on-site interactions along the synthetic dimension by pulsing strong intraspin interactions $U_{m m}$ in a successive and time-periodic manner; see Fig. 1(b). Specifically, one considers an experimental situation where one activates a strong interaction $U_{11}$ for a short duration $\tau$, then $U_{22}$ for the same duration $\tau$, etc., while keeping the background interactions [see Eq. (4) below] approximately constant and small. Physically, this requires enhancing the intraspin scattering lengths, one at a time, in a periodic manner. As we demonstrate below, this protocol allows one to investigate the Bose-Hubbard model in synthetic dimensions, under the condition that the individual scattering lengths are modified on sufficiently small time scales (compared with the physical time scales set by the effective Hubbard model).

\section{A. The Trotter sequence}

This protocol can be formulated in terms of a Trotter sequence [52]: Considering one cycle of the driving protocol, the time-evolution operator over one period $T \operatorname{reads}(\hbar=1$ in the following except where otherwise stated)

$$
\begin{aligned}
\hat{\mathcal{U}}(T) & =\hat{A}_{M}(\tau) \cdots \hat{A}_{m}(\tau) \cdots \hat{A}_{2}(\tau) \hat{A}_{1}(\tau), \\
\hat{A}_{m}(\tau) & =\exp \left(-i \tau \hat{H}_{m}\right),
\end{aligned}
$$

where

$$
\tau=T / M
$$

is the duration of each pulse and where the Hamiltonian at each step " $m$ " reads

$$
\hat{H}_{m}=-\sum_{n=1}^{M}\left(\Omega_{n} \hat{b}_{n+1}^{\dagger} \hat{b}_{n}+\text { H.c. }\right)+\frac{U}{2} \hat{n}_{m}\left(\hat{n}_{m}-1\right) .
$$

Hence, during the $m$ th step, the system is assumed to evolve under the presence of the intraspin interaction $U_{m m}=U$ only; we note that the scheme requires that the parameter $U$ should be independent of $m$. For the sake of simplicity, we have neglected the presence of the background (infinite-range) interactions, which at the $m$ th step read

$$
\begin{aligned}
\hat{H}_{m}^{\mathrm{bg}}= & \sum_{n \neq m} \frac{U_{n n}}{2} \hat{n}_{n}\left(\hat{n}_{n}-1\right)+\sum_{j, l \neq j} \frac{U_{j l}}{2} \hat{n}_{j} \hat{n}_{l} \\
& \text { with } U_{n n}, U_{j l} \ll U_{m m}=U .
\end{aligned}
$$

These effects will be discussed below in Sec. II B. Besides, we henceforth neglect the state dependence of the coupling matrix elements and set $\Omega_{n}=\Omega$.
The scheme builds on repeating the sequence in Eqs. (2) and (3) in a fast and time-periodic manner. In this case, the long-time evolution of the system is well captured by the effective (Floquet) Hamiltonian $\hat{H}_{F}$ defined through [52,53]

$$
e^{-i T \hat{H}_{F}} \equiv \hat{\mathcal{U}}(T) .
$$

In the high-frequency regime, where the drive frequency satisfies $\omega=2 \pi / T \gg(\Omega, U)$, the effective Hamiltonian $\hat{H}_{F}$ can be evaluated by applying the Trotter formula,

$$
e^{-i \tau \hat{H}_{1}} e^{-i \tau \hat{H}_{2}} \approx e^{-i \tau\left(\hat{H}_{1}+\hat{H}_{2}\right)},
$$

to the sequence in Eq. (2); we note that this approximation corresponds to the lowest order of the Magnus expansion [53], namely, to considering the time average of the time-dependent Hamiltonian describing the Trotter sequence over a single period $T$. Altogether, this Trotter approach yields

$$
\hat{\mathcal{U}}(T) \approx \exp \left(-i \tau \sum_{m=1}^{M} \hat{H}_{m}\right) \equiv e^{-i T \hat{H}_{\mathrm{TBH}}}, \quad \tau=T / M,
$$

which identifies the effective Hamiltonian as the 1D BoseHubbard Hamiltonian $\hat{H}_{\mathrm{TBH}}$ defined as

$$
\begin{aligned}
\hat{H}_{\mathrm{TBH}}= & -J_{\text {eff }} \sum_{m=1}^{M}\left(\hat{b}_{m}^{\dagger} \hat{b}_{m+1}+\hat{b}_{m+1}^{\dagger} \hat{b}_{m}\right) \\
& +\frac{U_{\text {eff }}}{2} \sum_{m} \hat{n}_{m}\left(\hat{n}_{m}-1\right),
\end{aligned}
$$

with the effective tunneling and interaction strengths

$$
J_{\text {eff }}=\Omega, \quad U_{\text {eff }}=U / M .
$$

Summarizing, pulsing strong intraspin interactions in a fast and time-periodic manner should allow one to investigate the physics of the Bose-Hubbard model in an atomic synthetic dimension, as captured by the effective "Trotter-Bose-Hubbard" Hamiltonian $\hat{H}_{\mathrm{TBH}}$ in Eq. (8).

\section{B. Effects of a residual infinite-range interaction}

As previously noticed, the effective Bose-Hubbard Hamiltonian in Eq. (8) was derived upon making the assumption that the background "infinite"-range repulsion in Eq. (4) can be neglected. In this section, we study the validity of such an approximation, by taking a finite background interaction into account during each step of the Trotter sequence.

For the sake of simplicity, we take this background interaction to be constant and set $U_{n n}=U_{j l}=V$ in Eq. (4). In this case, the perturbation can be written as

$$
\begin{aligned}
\hat{H}_{m}^{\text {bg }} & =\frac{V}{2} \sum_{n \neq m} \hat{n}_{n}\left(\hat{n}_{n}-1\right)+\frac{V}{2} \sum_{j, l \neq j} \hat{n}_{j} \hat{n}_{l} \\
& =\frac{V}{2} \sum_{j}\left(\hat{n}_{j}\left(\hat{n}_{j}-1\right)+\sum_{l \neq j} \hat{n}_{j} \hat{n}_{l}\right)-\frac{V}{2} \hat{n}_{m}\left(\hat{n}_{m}-1\right) \\
& =\text { const }-\frac{V}{2} \hat{n}_{m}\left(\hat{n}_{m}-1\right),
\end{aligned}
$$

where we used that the $\mathrm{SU}(M)$-invariant interaction term in the second line is constant (it only depends on the total particle 
number in the absence of other degrees of freedom). Hence this perturbation simply replaces $U \rightarrow(U-V)$ in Eq. (3), such that the effective Hamiltonian is still given by Eq. (8), now with the modified effective interaction strength $U_{\text {eff }}=$ $(U-V) / M$. In the following, we will neglect this effect by setting $V=0$ in our numerics.

The case of nonuniform background interactions can no longer be recast in the form of a renormalized interaction strength $U$. The study of the resulting perturbations, which depend on the precise values of the coupling constants, is beyond the scope of this work.

\section{EXPERIMENTAL IMPLEMENTATION}

The system discussed above can be implemented using a bosonic atom of hyperfine spin $F$, possessing $2 F+1$ levels $|m\rangle$, with $-F \leqslant m \leqslant F$. The coupling $\Omega_{m}$ between successive levels $m$ and $m+1$ would be induced using a two-photon Raman transition. Independent tuning of the $\Omega_{m}$ couplings can be achieved by applying a quadratic Zeeman shift, which lifts the degeneracy between the $m \leftrightarrow m+1$ transitions, allowing independent resonant couplings. Such a control over the coupling amplitudes can be used to vary the number of involved states $M \leqslant 2 F+1$.

In this paper, we explore the strongly interacting regime of the Trotter-Bose-Hubbard Hamiltonian in Eqs. (7) and (8), which corresponds to having a ratio $U_{\text {eff }} / J_{\text {eff }} \approx 1-10$. Realistic experimental values for the model parameters are $J_{\text {eff }}=\Omega \approx 2 \pi \times 100 \mathrm{~Hz}$ and $U=U_{\text {eff }} M \approx 2 \pi \times 1 \mathrm{kHz}$, which should allow for realistic observation times of a few hopping periods, $t \approx 1-10 \times\left(1 / J_{\text {eff }}\right)$, while limiting interaction-induced (three-body) losses.

For the proposed scheme, the various intraspin scattering lengths need to be dynamically tuned and modulated at a drive frequency $\omega \gg\left(J_{\text {eff }}, U_{\text {eff }}\right)$; see Sec. IV. Following the discussion above, this would correspond to having $\omega$ of the order of several kilohertz. We propose two possible schemes yielding to such a dynamical tuning of interactions.

Our main scheme described in Sec. II A could be implemented using multiple Fano-Feshbach resonances [48]. The scattering length $a_{m m}$ would be enhanced by switching the system close to a Feshbach resonance for collisions between atoms in the internal state $m$. This could be achieved by using standard magnetic tuning on the resonances, which would allow, if carefully implemented, for drive frequencies up to the order of the bandwidth of the magnetic field circuits (limited by the coils' inductance and eddy currents), typically a few tens of kilohertz [54,55]. In addition, the tuning of several scattering lengths via the magnetic field is intrinsically coupled, bringing additional corrections to the proposed scheme (see also Sec. II B). Better adapted tuning schemes can also be envisioned, such as schemes based on optical beams, radio-frequency fields, or microwave radiation [56-60]. Such schemes not only are intrinsically fast but also allow for an independent and simultaneous tuning of several scattering lengths. The control of multiple Feshbach resonances in spin mixtures was first proposed in Ref. [49] and was demonstrated experimentally with ${ }^{87} \mathrm{Rb}$ atoms in Ref. [50].

An alternative protocol could be based on a set of $M$ internal states, with a dominant scattering length $a_{11} \gg\left|a_{m n}\right|$,
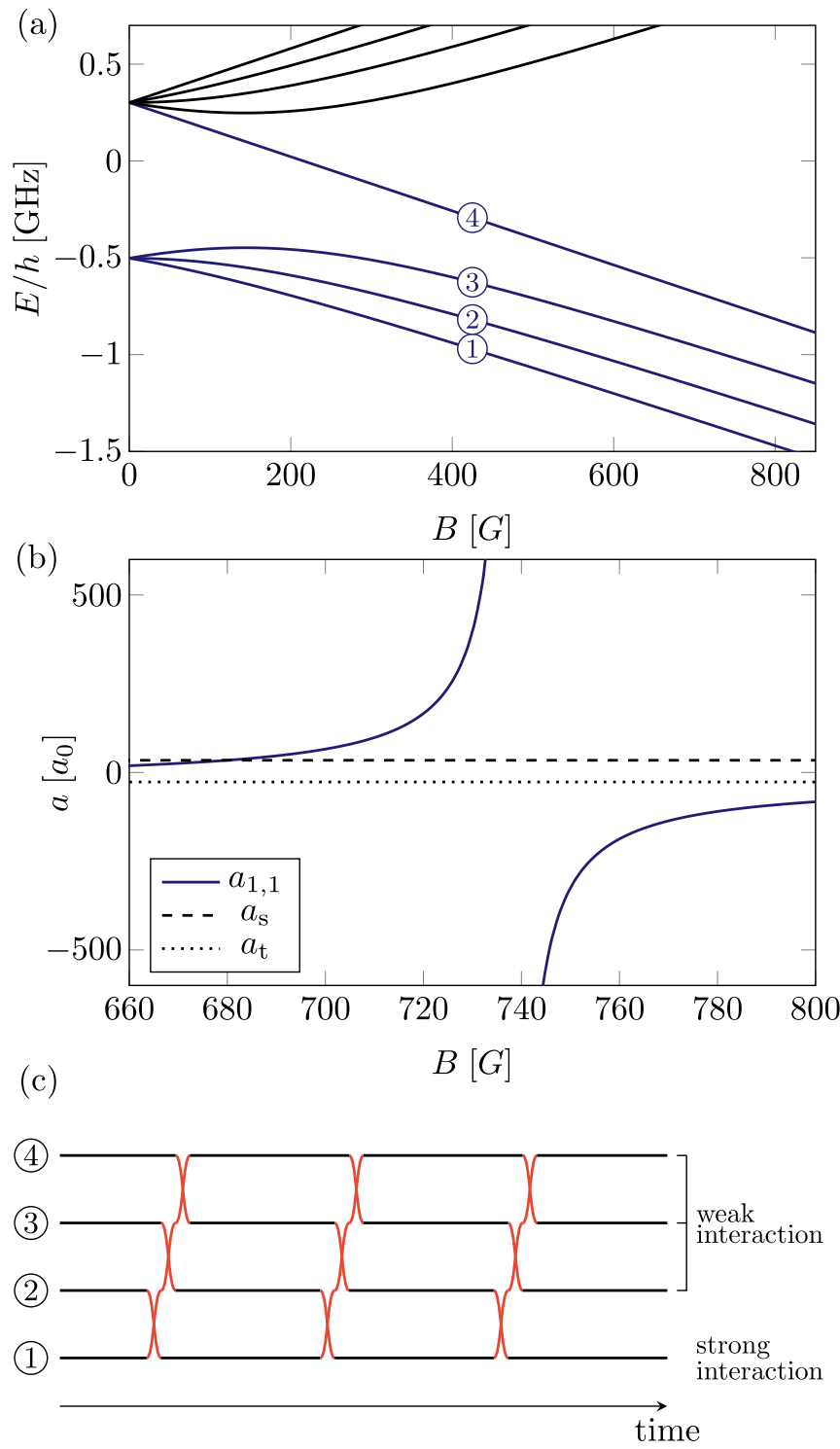

FIG. 2. (a) Structure of hyperfine (Zeeman) levels of ${ }^{7} \mathrm{Li}$ atoms. We propose to use the four lowest Zeeman states as a synthetic dimension of size $M=4$. (b) Variation of the scattering length $a_{1,1}$ with the magnetic field, exhibiting a Feshbach resonance at $B=738$ G. The singlet and triplet scattering lengths $a_{\mathrm{s}}$ and $a_{\mathrm{t}}$ are shown as dashed and dotted lines, respectively. (c) Scheme of the permutation between internal states used to engineer an effective interaction $U_{\text {eff }}=U_{11} / M$ between atoms of identical internal state only. The red curves indicate the exchange of a pair of spin states using a resonant radio-frequency $\pi$ pulse.

with $(m, n) \neq(1,1)$, such that one only keeps the interaction term $U_{11}$ in Eq. (2). In this setting, instead of dynamically tuning the interactions, one would then apply a series of fast radio-frequency pulses to drive a cyclic permutation $(1,2, \ldots, M) \rightarrow(M, 1, \ldots, M-1)$; see Fig. 2(c). After such a unitary transform, the system is described by the Hamiltonian

$$
\hat{H}=-\sum_{m=1}^{M}\left(\Omega_{m-1} \hat{b}_{m+1}^{\dagger} \hat{b}_{m}+\text { H.c. }\right)+\frac{U_{11}}{2} \hat{n}_{2}\left(\hat{n}_{2}-1\right) .
$$


If the couplings $\Omega_{m}$ are not uniform, they should also be changed according to the permutation $\Omega_{m-1} \rightarrow \Omega_{m}$, leading to the Hamiltonian

$$
\hat{H}=-\sum_{m=1}^{M}\left(\Omega_{m} \hat{b}_{m+1}^{\dagger} \hat{b}_{m}+\text { H.c. }\right)+\frac{U_{11}}{2} \hat{n}_{2}\left(\hat{n}_{2}-1\right) .
$$

Repeating this procedure $p$ times leads to the Hamiltonian

$$
\hat{H}=-\sum_{m=1}^{M}\left(\Omega_{m} \hat{b}_{m+1}^{\dagger} \hat{b}_{m}+\text { H.c. }\right)+\frac{U_{11}}{2} \hat{n}_{p}\left(\hat{n}_{p}-1\right),
$$

which features a dominant interaction between atoms in internal state $m=p$. By applying the cycles periodically in time, one obtains an effective Hamiltonian with an average interaction between identical internal states $m$ only, namely,

$$
\hat{H}_{\mathrm{eff}}=-\sum_{m=1}^{M}\left(\Omega_{m} \hat{b}_{m+1}^{\dagger} \hat{b}_{m}+\text { H.c. }\right)+\frac{U_{\mathrm{eff}}}{2} \sum_{m=1}^{M} \hat{n}_{m}\left(\hat{n}_{m}-1\right),
$$

where $U_{\text {eff }}=U_{11} / M$.

This protocol could be implemented with ${ }^{7} \mathrm{Li}$ atoms, using the $M=4$ hyperfine levels of lowest energy; see Fig. 2(a). The interaction between atoms in the ground state $|1\rangle$ can be tuned using a magnetic Feshbach resonance at $B=738 \mathrm{G}$. For a magnetic field $B \approx 733 \mathrm{G}$, the scattering length $a_{11} \simeq 316 a_{0}$ is much larger than the others, which are not resonant and thus remain bounded between the singlet and triplet scattering lengths $a_{\mathrm{s}}$ and $a_{\mathrm{t}}$, i.e., $\left|a_{m n}\right|<30 a_{0}$; see Fig. 2(b) and Ref. [61]. For this magnetic field, the cyclic permutation can be performed using radio-frequency pulses that are resonant with the $(1,2),(2,3)$, and $(3,4)$ transitions; see Fig. 2(c). The protocol can be extended to larger $M$ values using lanthanide atoms, which feature a large-size electronic spin.

Finally, we note that losses and heating can be of different nature in the present framework. A first loss mechanism is provided by two-body inelastic scattering. Spin-dependent $s$-wave scattering or magnetic dipolar interactions can in principle trigger spin-changing processes, such as the exothermic scattering $1+3 \rightarrow 2+2$. These processes depend on the atomic species and the experimental protocol used. For the case illustrated in Fig. 2, these processes are strongly inhibited in the considered regime of high magnetic fields (PaschenBack regime), for which the states $|m\rangle(m=1, \ldots, 4)$ are almost polarized in the same electronic state $\left|m_{S}=-1 / 2\right\rangle$, and differ by the nuclear spin projection $m_{I}=m-m_{S}$ only. Since the nuclear spin is essentially decoupled from atom-atom scattering, we expect a strong reduction of spinchanging inelastic processes, as verified in ultracold lithium mixtures [62]. A second loss channel is provided by inelastic three-body collisions, which can be enhanced upon tuning the scattering lengths. As indicated above, this effect can be limited by considering a small interaction parameter $U$, hence constraining the values of the scattering lengths as well as the particle density in the harmonic-oscillator ground state. Finally, while the Raman coupling can potentially lead to heating, we point out that these effects can be limited in realistic configurations $[12,17]$. We point out that these difficulties, which potentially affect any cold-atom realization of an internal-state synthetic dimension, should be analyzed on a case-by-case basis as they strongly depend on the actual implementation.

\section{NUMERICAL INVESTIGATIONS}

The next sections analyze the validity and applicability of the scheme described in Sec. II A, in the relevant situation of a small ensemble of $N$ atoms. In order to explore the physics of the Bose-Hubbard model at unit filling $(\bar{n}=1)$, we will henceforth set $N=M$, where $M$ corresponds to the number of available internal states, i.e., the number of sites along the synthetic dimension. As reminded above, the number of involved internal states can be adjusted by controlling individual couplings, and it can thus be made even or odd.

The emblematic Bose-Hubbard model has played a key role in our exploration of quantum matter, by providing a minimal setting by which a genuine quantum phase transition can be observed: the transition from a superfluid to a Mott insulating state [63-66]. At unit filling, the Mott state is characterized by the occupation of each site by one particle; this feature is signaled by a small average value of the operator

$$
\hat{n}_{D}=\frac{1}{N} \sum_{m} \frac{\hat{n}_{m}\left(\hat{n}_{m}-1\right)}{2},
$$

which counts the average number of doubly occupied sites. Besides, the Mott state is also associated with a nonlocal "parity" parameter $[67,68]$, which signals the appearance of correlated particle-hole pairs, as captured by the average value of the operator [68]

$$
\hat{O}_{p}(l)=e^{i \pi \sum_{m<l}\left(\hat{n}_{m}-\bar{n}\right)},
$$

where $\bar{n}=1$ denotes the average background density and $l$ denotes a generic lattice site. In contrast, the superfluid phase is characterized by a vanishing gap (in the thermodynamic limit), a significant number of doubly occupied sites, and a zero parity parameter $\left\langle\hat{O}_{p}(l)\right\rangle$. At unit filling $(\bar{n}=1)$, the ground state of the Bose-Hubbard Hamiltonian in Eq. (8) forms a Mott state when $U_{\text {eff }} \gtrsim 3 J_{\text {eff }}$ and a superfluid otherwise; see Refs. [69-71].

\section{A. Accuracy of the Trotter-Bose-Hubbard Hamiltonian}

In the present Trotter approach, we expect that accuracy is reached in the limit $\tau \rightarrow 0$, namely, when the frequency of the periodic drive $\omega$ substantially exceeds all other frequencies in the system: As $\hbar \omega$ becomes comparable to other energy scales (as set by the model parameters $U_{\text {eff }}$ and $J_{\text {eff }}$ ), one expects corrections to the "ideal" (Trotter) Hamiltonian in Eq. (8) to be significant $[52,72]$.

As a first step, we explore the accuracy of the Trotter-BoseHubbard Hamiltonian in Eq. (8) by performing numerical [time-dependent density-matrix renormalization group (tDMRG)] simulations [73,74] of the dynamical evolution of a system of $N=M$ atoms under the full time-dependent sequence in Eqs. (2) and (3). In these simulations, the initial state $\left|\psi_{0}\right\rangle=\left|\phi_{\mathrm{TBH}}^{\mathrm{GS}}\right\rangle$ is taken to be the ground state of the Trotter-Bose-Hubbard Hamiltonian $\hat{H}_{\mathrm{TBH}}$ in Eq. (8), and the time-evolution operator associated with the multistep sequence in Eqs. (2) and (3) is applied in a repeated manner at the frequency $\omega$. From these simulations, we characterize the 
deviation of the full Trotter dynamics from the ideal dynamics associated with the effective Bose-Hubbard Hamiltonian in Eq. (8). In our study, these deviations (or "Trotter errors") are evaluated by considering the expectation values of both the local double-occupancy observable in Eq. (15) and the nonlocal "parity" observable in Eq. (16), namely, by determining

$$
\begin{gathered}
\Delta n_{D}(t)=\left|n_{D}(t)-n_{D}^{\text {ideal }}\right|, \\
\Delta O_{p}(t)=\left|O_{p}(t, l)-O_{p}^{\text {ideal }}\right|,
\end{gathered}
$$

where the ideal quantities correspond to the expectation value of these operators in the ground state of the effective Hamiltonian in Eq. (8); we note that in our simulations, these ideal values correspond to the expectation values at time $t=0$. In these calculations, we set $U_{\text {eff }} / J_{\text {eff }}=1$.

We point out that our study of deviations between the Trotter and the targeted dynamics is inspired by Ref. [72], which identified the existence of two radically different regimes as one varies the time step $\tau$ of a Trotter sequence. In the limit of large $\tau$ (i.e., away from the high-frequency regime), the system is in a "delocalized quantum chaotic regime" where an initially prepared state is scrambled across the full basis of Floquet eigenstates, hence leading to substantial and uncontrolled deviations from the desired dynamics of local observables. In contrast, for sufficiently small $\tau$ (i.e., in the high-frequency regime), the system can enter a "localized" regime, where the dynamics remains constrained over long time scales and where the Trotter error associated with local observables exhibits a characteristic quadratic dependence in the time step $\tau$. It is the aim of this section to analyze the existence of a localized, and hence operational, regime in our setting.

Our numerical results are summarized in Fig. 3. For a sufficiently large drive frequency (here, $\omega \gtrsim 10 \pi J_{\mathrm{eff}}$ ), both observables in Eqs. (17) and (18) undergo a small micromotion around the initial (ideal) value. This controlled Trotter error, which remains small over long time scales, is further validated in the inset of Fig. 3(a), which shows the characteristic quadratic growth of the error $\Delta n_{D}(t)$ as a function of the time step $\tau$. Following Ref. [72] and the discussion above, these results confirm the existence of a localized regime, which reflects the high fidelity of the initially prepared (ideal) eigenstate $\left|\phi_{\mathrm{TBH}}^{\mathrm{GS}}\right\rangle$ with respect to the time-evolved state. We also point out that a similar behavior was found for the nonlocal observable in Eq. (18).

In contrast, for a smaller frequency $\omega \lesssim 5 \pi J_{\text {eff }}$, one observes substantial deviations from the ideal expectation values in the form of a larger micromotion, as well as a dynamical increase in the Trotter error (see orange curves in Fig. 3).

Altogether, these studies offer a first confirmation that the Trotter sequence in Eqs. (2) and (3) indeed effectively reproduces the time dynamics associated with the Bose-Hubbard Hamiltonian, offering a viable scheme by which the latter model could be explored in an atomic synthetic dimension.

\section{B. From the superfluid to the Mott state in an atomic synthetic dimension}

The accuracy between the real-time evolution associated with the Trotter sequence [Eqs. (2) and (3)] and that
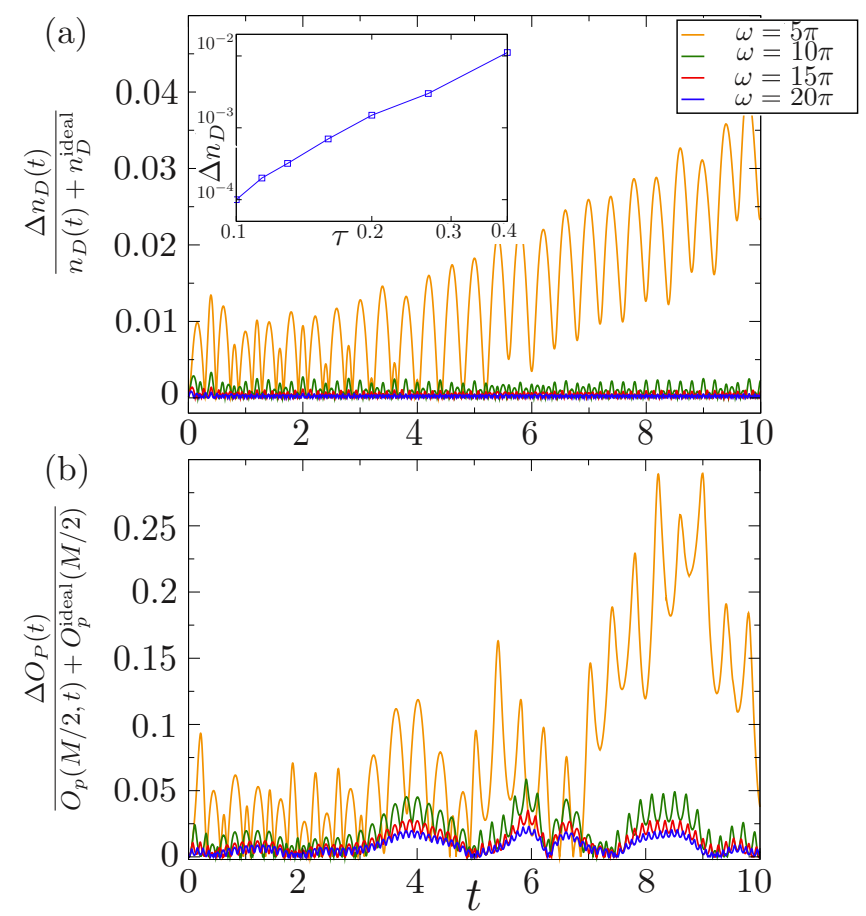

FIG. 3. Time evolution of the renormalized (a) $\Delta n_{D}$ and (b) $\Delta O_{p}$ calculated in $l=M / 2$ for fixed $M=N=10$ and $U_{\text {eff }} / J_{\text {eff }}=1.0$ and different values of $\omega$ (in units of $J_{\text {eff }}$ ). The inset in (a) shows the quadratic growth of $\Delta n_{D}$ for different periods. The times $t$ and $\tau$ are expressed in units of $1 / J_{\text {eff }}$, and the frequency $\omega$ is expressed in units of $J_{\text {eff. }}$.

associated with the target (ideal) Bose-Hubbard Hamiltonian in Eq. (8) was previously confirmed in the superfluid regime corresponding to $U_{\text {eff }}=J_{\text {eff }}$. We now validate this agreement through the superfluid-to-Mott transition, upon increasing the effective interaction strength. In our numerics, we evaluate the local and nonlocal observables that were previously introduced in Eqs. (15) and (16), as well as the compressibility defined as

$$
\Delta n(t)=\frac{1}{M} \sum_{m}\left(\left\langle\hat{n}_{m}^{2}\right\rangle-\left\langle\hat{n}_{m}\right\rangle^{2}\right),
$$

which provides a further probe to distinguish between the two competing phases: In the thermodynamic limit, the superfluid phase is gapless (compressible), while the Mott state is gapped (incompressible). Figure 4 shows the time evolution of these three observables, when preparing the initial state in the ground state of the effective Hamiltonian and acting on this state with the Trotter sequence. Importantly, we observe that all three observables undergo a very small micromotion around their ideal (initial) value, for all values of the ratio $U_{\text {eff }} / J_{\text {eff }}$ considered. While the transition from the superfluid to the Mott state does not show a sharp behavior, which is indeed expected in such a small system size $(M=10)$, one does recognize the main features of this quantum phase transition: Indeed, both the double-occupancy $n_{D}(t)$ and the compressibility $\Delta n(t)$ show smaller values upon crossing the expected transition point $\left(U_{\text {eff }} / J_{\text {eff }} \gtrsim 3\right)$, while the parity parameter $O_{p}(t)$ shows an opposite behavior (i.e., significantly larger values are obtained in the Mott regime). We point out 

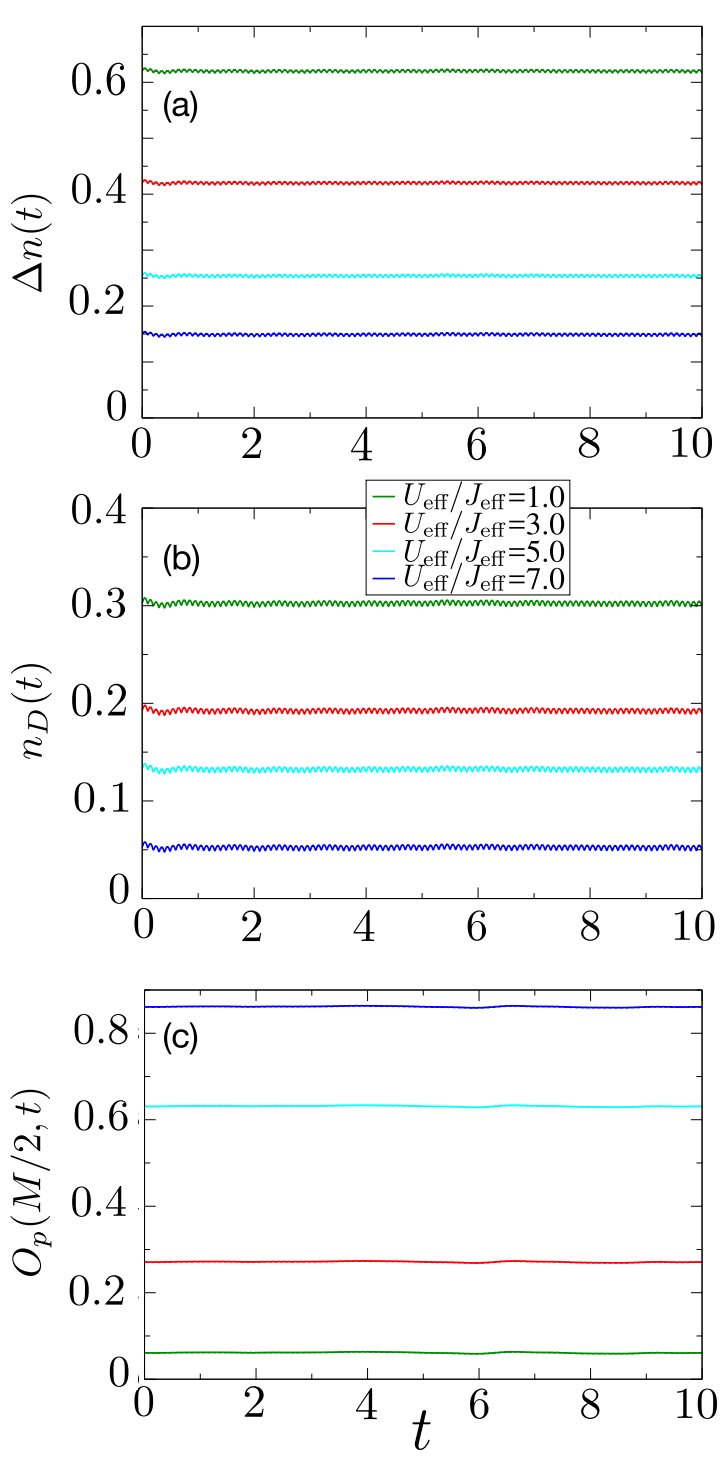

FIG. 4. Time evolution of (a) $\Delta n(t)$, (b) $n_{D}(t)$, and (c) $O_{p}(M / 2, t)$ for fixed $M=N=10$ and $\omega=20 \pi J_{\text {eff }}$ and different values of $U_{\text {eff }} / J_{\text {eff }}$. The time $t$ is expressed in units of $1 / J_{\text {eff }}$.

that the sharpness of this superfluid-to-Mott transition can be improved by considering more internal states $(M>10)$, i.e., a longer synthetic dimension.

\section{The flux ladder configuration: Combining a synthetic dimension with a double well}

In this section, we explore the possibility of extending the dimensionality of the effective Bose-Hubbard model in Eq. (8), by considering atoms trapped in a double-well potential. Upon taking the synthetic dimension into account, this configuration leads to a fictitious ladder geometry that is aligned along the synthetic dimension: Hopping from a well to the other corresponds to hopping on the rungs of the ladder, while hopping along the internal-state (synthetic) dimension corresponds to moving along the legs of the ladder. As already pointed out in Ref. [5], the hopping matrix elements along the synthetic dimension are naturally associated with complex phase factors (which are attributed to the phases of the Raman lasers), which in turn generates synthetic magnetic fluxes in each plaquette of the ladder.

Applying the Trotter sequence in Eqs. (2) and (3) to this double-well setting leads to the effective Hamiltonian

$$
\begin{aligned}
\hat{H}= & -J_{\text {eff }} \sum_{m, \sigma}\left(e^{i \theta \sigma} \hat{b}_{m, \sigma}^{\dagger} \hat{b}_{m+1, \sigma}+\text { H.c. }\right) \\
& -\frac{J_{\text {real }}}{2} \sum_{m}\left(\hat{b}_{m, \frac{1}{2}}^{\dagger} \hat{b}_{m,-\frac{1}{2}}+\text { H.c. }\right) \\
& +\frac{U_{\text {eff }}}{2} \sum_{m, \sigma} \hat{n}_{m, \sigma}\left(\hat{n}_{m, \sigma}-1\right),
\end{aligned}
$$

where we have again neglected the residual background infinite-range interaction in Eq. (4). Here, in addition to the parameters $J_{\text {eff }}$ and $U_{\text {eff }}$ already defined in Eq. (8), the Hamiltonian includes the parameter $J_{\text {real }}$ that describes tunneling between the two wells (which we label as $\sigma= \pm 1 / 2$ ). The hopping matrix elements along the synthetic dimension also contain a phase factor $\exp ( \pm i \theta / 2)$, which explicitly depends on the well (or the leg of the ladder) $\sigma$. We note that a similar synthetic ladder configuration was realized using two longlived electronic states of fermionic ytterbium atoms [12], in which case the long (leg) direction was associated with a real (optical) lattice.

The bosonic ladder Hamiltonian in Eq. (20) has a rich ground-state phase diagram, featuring various types of socalled Meissner and vortex states [75-80], which can be identified by evaluating the currents associated with the leg (synthetic) and rung (real) directions. In the following, we study the existence of such phases in the case of a particle density $\bar{n}=N /(2 M)=1 / 2$ in the synthetic ladder. Following Ref. [80], we analyze the "chiral" current $\hat{\mathcal{J}}_{c}$ and the local "rung" current $\hat{\mathcal{J}}_{r}$ defined as

$$
\begin{aligned}
& \hat{\mathcal{J}}_{c}=-i J_{\text {eff }} \sum_{m, \sigma}\left(\sigma e^{i \theta \sigma} \hat{b}_{m, \sigma}^{\dagger} \hat{b}_{m+1, \sigma}-\text { H.c. }\right), \\
& \hat{\mathcal{J}}_{r}=-\frac{i J_{\text {real }}}{M} \sum_{m \in \text { center }}\left(\hat{b}_{m, \frac{1}{2}}^{\dagger} \hat{b}_{m,-\frac{1}{2}}-\text { H.c. }\right),
\end{aligned}
$$

where " $m \in$ center" refers to a few selected "sites" around the central "site" $m=M / 2$. We point out that the chiral-current operator associates different flow directions with the two legs of the ladder $(\sigma)$, hence probing the orientation (or chirality) of the flow taking place along the edge of the ladder. In contrast, the local rung-current operator probes vortices in the ladder, which are associated with flows taking place around a few inner plaquettes; see Refs. [76-80].

In the Mott regime, the Meissner-type phase is characterized by a large chiral current and by a vanishing rung current: This phase is associated with a clear chiral motion along the legs of the ladder. In contrast, the Mott-vortex phase displays a significant rung current accompanied with a reversal of the chiral current; see Fig. 5 in Ref. [80].

We demonstrate in Fig. 5 that such phases are indeed supported by the interacting synthetic ladder generated by the Trotter sequence in Eqs. (2) and (3). This figure shows the time evolution of the chiral and rung currents, when preparing the initial state in the ground state of the effective Hamiltonian in Eq. (20) and acting on this state with the 


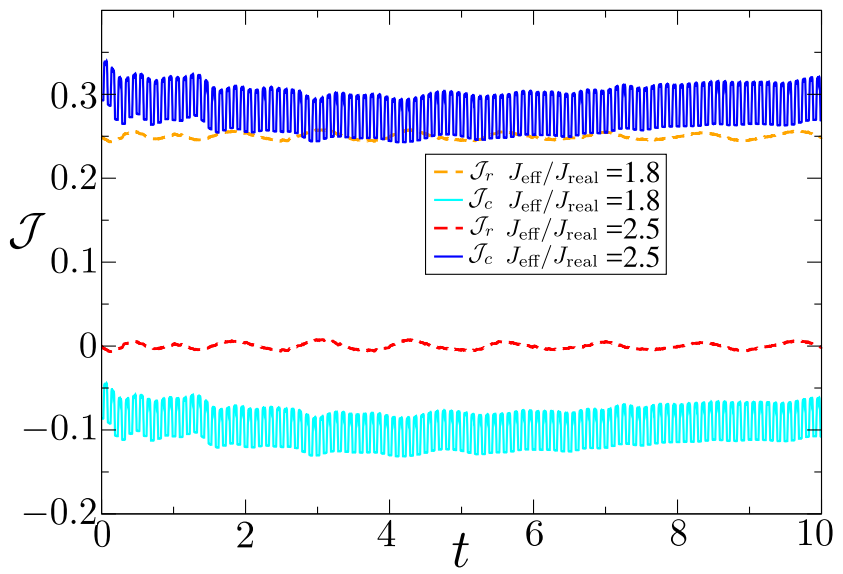

FIG. 5. Time evolution of the rung current $\mathcal{J}_{r}$ and chiral current $\mathcal{J}_{c}$ for a synthetic ladder with a density $N / 2 M=0.5$ with $M=N=$ $8, \omega=20 \pi J_{\text {eff }}, \theta=0.9 \pi$, and $U_{\text {eff }} / J_{\text {eff }}=4$ and different values of $J_{\text {real }} / J_{\text {eff. }}$ The local rung current is evaluated using the four central sites. The time $t$ is expressed in units of $1 / J_{\text {eff }}$, the currents are expressed in units of $J_{\text {eff }}$, and the chosen initial state corresponds to the ground state of Eq. (20).

Trotter sequence. As in the previous examples, one observes a very weak micromotion of these observables around their ideal (initial) value, which coincides with those previously reported in Ref. [80]: Depending on the value of $J_{\text {real }} / J_{\text {eff }}$, the states have large (weak) mean chiral (rung) current or a large rung current accompanied with a weak and negative chiral current.

These results clearly show that both types of phases (Meissner and vortex) are indeed supported by the Trotterengineered interacting ladder, in the Mott regime $\left(U_{\text {eff }} / J_{\text {eff }}>\right.$ $3)$. An interesting perspective concerns the realization of Laughlin-type states (fractional Chern insulators) in this setting [81], by extending the size of the "real" direction.

\section{Quench dynamics and magnetic order in a synthetic dimension}

The previous sections explored the ground-state properties of the effective Trotter-Bose-Hubbard Hamiltonian [Eq. (8)]. Specifically, those sections analyzed the time evolution of a specific initial state, taken to be the ground state of the effective Hamiltonian, upon applying the Trotter sequence in Eqs. (2) and (3). In this last section, we now demonstrate that this interacting synthetic-dimension setting is also well suited to explore intriguing quench dynamics, namely, situations where the initial state does not correspond to an eigenstate of the effective Hamiltonian.

Of particular interest are quench dynamics involving dynamically formed bound states-so-called bound pairs (BPs) in the case of contact interactions-which can be realized in ultracold atoms through a special state preparation. Let us first recall the basic principles behind this concept: Consider a pair of atoms initially occupying a single lattice site; the energy difference between this configuration and that corresponding to spatially separated atoms is then simply given by the onsite (Hubbard) interaction. When the latter is strong enough (irrespective of its sign), energy conservation forces the pair to remain bound: The pair is forbidden to decay by converting its potential energy into kinetic energy due to the fact that the latter is restricted by the bandwidth of the populated Bloch band. In this regime, the prepared pair moves over the lattice, with both atoms tunneling together to the same neighboring sites. Such scenarios were theoretically proposed to create effective antiferromagnetic states [82], entangled Bell pairs [83], and metastable fermionic superfluids [84]. On the experimental side, such quench dynamics allowed for the observation of repulsive BPs [85,86] and magnon dynamics [87]. We also note that similar scenarios can arise in the regime of attractive interactions [88].

It is the aim of this section to analyze the quench dynamics of bound pairs in atomic synthetic dimensions with engineered on-site interactions [Eqs. (2) and (3)]. To this purpose, we consider an initial state of the form

$$
|\psi(t=0)\rangle=\left|n_{1}, n_{2}, \ldots, n_{M}\right\rangle=|0,2,0,2, \ldots, 0,2\rangle,
$$

where $n_{m}$ corresponds to the (initial) number of atoms in the internal state $m$. This initial configuration corresponds to having a (synthetic) lattice with alternating doubly occupied or empty lattice sites (with no single occupancy). We consider acting on this initial state with the Trotter sequence in Eqs. (2) and (3), which was previously shown to mimic the time evolution associated with the effective Bose-Hubbard Hamiltonian in Eq. (8). In this section, we also set the interactions to be attractive [88], i.e., $U_{\text {eff }}<0$. In this configuration, we expect the initial pairs (in internal states with $m$ even) to remain bound in the synthetic dimension for sufficiently strong interactions $\left|U_{\text {eff }} / J_{\text {eff }}\right| \gg 1$ and to "tunnel" to neighboring sites $m$ with an effective hopping matrix element smaller than the single-particle hopping matrix element $J_{\text {eff }}$. We point out that the number of particles "per site" is not truncated in our simulations: The fact that the number of pairs and empty sites is conserved during the evolution is simply due to the energetic constraint imposed by the strong "on-site" interaction.

In this configuration where attractive bound pairs are formed and no single occupation is present, the system is captured by an effective model [82]

$$
\hat{H}=-J_{\mathrm{BP}} \sum_{m}\left(\hat{c}_{m}^{\dagger} \hat{c}_{m+1}+\text { H.c. }\right)+B \sum_{m} \hat{c}_{m}^{\dagger} \hat{c}_{m+1}^{\dagger} \hat{c}_{m+1} \hat{c}_{m},
$$

where $\hat{c}_{m}^{\dagger}$ creates a pair of atoms (a hard-core boson) in the $m$ th internal state, where $J_{\mathrm{BP}}=-2 J_{\text {eff }}^{2} / U_{\text {eff }}$ is the "nearest-neighbor" pair hopping, and where $B=-16 J_{\text {eff }}^{2} / U_{\text {eff }}$ describes the density-density interaction strength between two neighboring pairs. Interestingly, a simple transformation $\hat{S}_{m}^{z}=$ $\left(1-\hat{n}_{m}\right) / 2$ maps this Hamiltonian onto a spin chain reminiscent of the XXZ model. In particular, for $B / J_{\mathrm{BP}}>1$, the system is known to undergo a phase transition from a paramagnetic to an antiferromagnetic state; in terms of the original bosons, this corresponds to a transition from a superfluid to a charge density wave.

We now demonstrate that our interacting synthetic dimension supports dynamically induced antiferromagnetic states of bound pairs. To do so, we consider the initial state in 


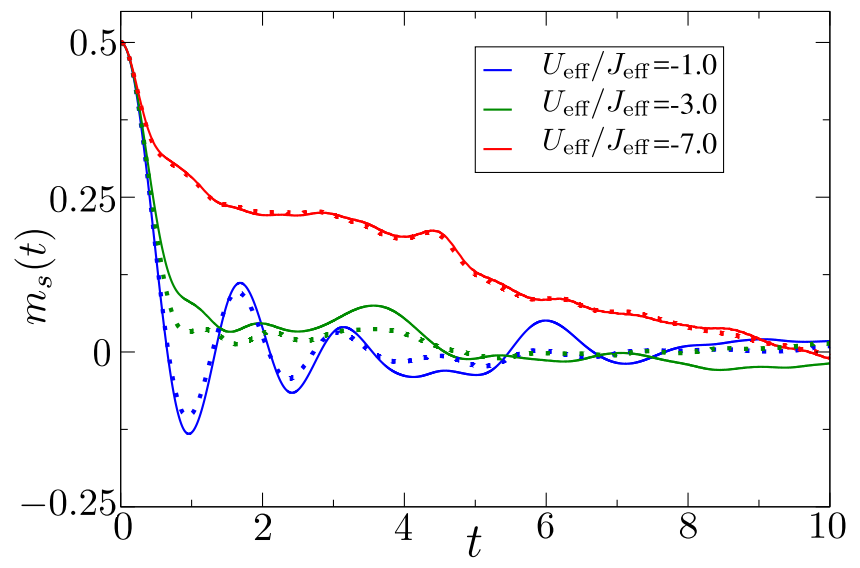

FIG. 6. Time evolution of the staggered magnetization $m_{s}$ for different values of $U_{\text {eff }} / J_{\text {eff }}$. In all the results we fixed $M=N=8$ and $\omega=20 \pi J_{\text {eff }}$, and the time $t$ is expressed in units of $1 / J_{\text {eff }}$. The chosen initial state corresponds, $\left|n_{1}, n_{2}, \ldots, n_{M}\right\rangle=|0,2,0,2, \ldots, 0,2\rangle$; see Eq. (23). Solid curves show the dynamics associated with the Trotter sequence, while dotted curves show that generated by the effective Bose-Hubbard Hamiltonian in Eq. (8), using the same initial state.

Eq. (23) and monitor the time evolution of the mean staggered magnetization

$$
\hat{m}_{s}=\frac{1}{N} \sum_{m}(-1)^{m}\left(\hat{n}_{m}-1\right),
$$

under the Trotter sequence [Eqs. (2) and (3)]. In the spin-1/2 representation, the quantity $\left\langle\hat{m}_{s}\right\rangle$ acts as an order parameter for the antiferromagnetic state, while it detects a density wave state in terms of the original bosons. Following Ref. [89], we expect the staggered magnetization to display oscillations around the value $m_{s}=0$ for a small ratio $B / J_{\mathrm{BP}}$, the system effectively behaving as free fermions in this paramagnetic regime. In contrast, for $B / J_{\mathrm{BP}}>1$, one expects $m_{s}$ to decay exponentially to zero, as a signature of the underlying antiferromagnetic order [89]. This behavior is precisely recovered in our numerical results displayed in Fig. 6, which shows the time evolution of the staggered magnetization for various values of the ratio $U_{\text {eff }} / J_{\text {eff }}$. For sufficiently strong intraspin interactions, the system dynamically forms bound pairs in the synthetic dimension, hence leading to an underlying magnetic order and an approximately exponential decay of the staggered magnetization over time as captured by the effective Hamiltonian in Eq. (24). We point out that a comparison between the time evolution associated with the Trotter sequence (solid curves in Fig. 6) and the dynamics generated by the effective Bose-Hubbard Hamiltonian in Eq. (8) (dotted curves in Fig. 6), as obtained from the same initial state [Eq. (23)], shows excellent agreement. We finally note that these results are compatible with those presented in Ref. [89].

These results confirm the viability of our scheme in view of observing dynamically formed bound states and artificial magnetism using atomic synthetic dimensions.

\section{CONCLUDING REMARKS}

This work introduced a realistic scheme by which strong and uniform "on-site" interactions can be generated and controlled in synthetic dimensions based on atomic internal states. This novel feature significantly broadens the applicability of synthetic dimensions in view of realizing and observing strongly interacting states of matter in ultracold gases.

In this proposal, we have illustrated and validated this scheme using several examples, including the study of unusual phases in strongly interacting ladders with flux and signatures of antiferromagnetic order upon a quench. Our numerical simulations, based on t-DMRG, confirm that these appealing phases and dynamics could indeed be explored in small atomic synthetic dimensions, using available technologies.

An interesting perspective concerns the extension of the 1D Bose-Hubbard model treated in this paper to higherdimensional settings, which could be realized by combining an atomic synthetic dimension with a real dimension (i.e., an optical lattice) or another synthetic dimension spanned by a secondary internal degree of freedom. This possibility, together with the engineered interactions introduced in this paper, would offer a new scenario by which the physics of 2D Hubbard models (including fractional Chern insulators) could be studied in ultracold atoms. Another important direction concerns the realization of the Fermi Hubbard model in synthetic dimensions, using a generalization of the present scheme.

Finally, a possible extension of this work would be the implementation of two-body "imaginary" interactions, by coupling the magnetic sublevels to molecular states in excited electronic states. Such photoassociation couplings can be internal-state dependent and could be applied continuously on the atomic sample [90].

\section{ACKNOWLEDGMENTS}

We thank J. Budich, J. Dalibard, F. Ferlaino, P. Hauke, R. Lopes, T. Scaffidi, L. Tarruell, and P. Zoller for useful discussions. We also thank B. Gadway for suggesting that "imaginary" interactions could also be implemented in this context through photoassociation couplings. Work in Brussels is supported by the FRS-FNRS and the ERC through the Starting Grant project TopoCold. L.C. acknowledges the support of the DFG-FWF (Austrian Science Fund) via FOR Grant No. FOR2247/PI2790. S.N. acknowledges support from the European Union through the ERC Grant projects UQUAM and TOPODY. L.B. acknowledges support from Spanish Ministry of Economy and Competitiveness [Severo Ochoa program for Centres of Excellence in R\&D (CEX2019-000910-S)], Plan National FISICATEAMO, Fundació Privada Cellex, Fundació Mir-Puig, and Generalitat de Catalunya (AGAUR Grant No. 2017 SGR 1341, CERCA program, and QuantumCAT U16-011424, cofunded by ERDF Operational Program of Catalonia 2014-2020). L.C. acknowledges support from the FWF (Austrian Science Fund) via the Elise Richter Fellowship, Grant No. V792. 
[1] I. M. Georgescu, S. Ashhab, and F. Nori, Quantum simulation, Rev. Mod. Phys. 86, 153 (2014).

[2] I. Bloch, J. Dalibard, and W. Zwerger, Many-body physics with ultracold gases, Rev. Mod. Phys. 80, 885 (2008).

[3] P. Windpassinger and K. Sengstock, Engineering novel optical lattices, Rep. Prog. Phys. 76, 086401 (2013).

[4] O. Boada, A. Celi, M. Lewenstein, and J. I. Latorre, Quantum Simulation of an Extra Dimension, Phys. Rev. Lett. 108, 133001 (2012).

[5] A. Celi, P. Massignan, J. Ruseckas, N. Goldman, I. B. Spielman, G. Juzeliunas, and M. Lewenstein, Synthetic Gauge Fields in Synthetic Dimensions, Phys. Rev. Lett. 112, 043001 (2014).

[6] T. Ozawa and H. M. Price, Topological quantum matter in synthetic dimensions, Nat. Rev. Phys. 1, 349 (2019).

[7] G. Salerno, H. M. Price, M. Lebrat, S. Häusler, T. Esslinger, L. Corman, J.-P. Brantut, and N. Goldman, Quantized Hall Conductance of a Single Atomic Wire: A Proposal Based on Synthetic Dimensions, Phys. Rev. X 9, 041001 (2019).

[8] B. Bauer, T. Pereg-Barnea, T. Karzig, M.-T. Rieder, G. Refael, E. Berg, and Y. Oreg, Topologically protected braiding in a single wire using Floquet Majorana modes, Phys. Rev. B 100, 041102(R) (2019).

[9] H. M. Price, O. Zilberberg, T. Ozawa, I. Carusotto, and N. Goldman, Four-Dimensional Quantum Hall Effect with Ultracold Atoms, Phys. Rev. Lett. 115, 195303 (2015).

[10] T. Ozawa, H. M. Price, N. Goldman, O. Zilberberg, and I. Carusotto, Synthetic dimensions in integrated photonics: From optical isolation to four-dimensional quantum Hall physics, Phys. Rev. A 93, 043827 (2016).

[11] I. Petrides, H. M. Price, and O. Zilberberg, Six-dimensional quantum Hall effect and three-dimensional topological pumps, Phys. Rev. B 98, 125431 (2018).

[12] L. F. Livi, G. Cappellini, M. Diem, L. Franchi, C. Clivati, M. Frittelli, F. Levi, D. Calonico, J. Catani, M. Inguscio, and L. Fallani, Synthetic Dimensions and Spin-Orbit Coupling with an Optical Clock Transition, Phys. Rev. Lett. 117, 220401 (2016).

[13] S. Kolkowitz, S. L. Bromley, T. Bothwell, M. L. Wall, G. E. Marti, A. P. Koller, X. Zhang, A. M. Rey, and J. Ye, Spin-orbitcoupled fermions in an optical lattice clock, Nature (London) 542, 66 (2017).

[14] B. Sundar, B. Gadway, and K. R. Hazzard, Synthetic dimensions in ultracold polar molecules, Sci. Rep. 8, 3422 (2018).

[15] B. Gadway, Atom-optics approach to studying transport phenomena, Phys. Rev. A 92, 043606 (2015).

[16] H. M. Price, T. Ozawa, and N. Goldman, Synthetic dimensions for cold atoms from shaking a harmonic trap, Phys. Rev. A 95, 023607 (2017).

[17] B. Stuhl, H.-I. Lu, L. Aycock, D. Genkina, and I. Spielman, Visualizing edge states with an atomic Bose gas in the quantum Hall regime, Science 349, 1514 (2015).

[18] F. A. An, E. J. Meier, and B. Gadway, Direct observation of chiral currents and magnetic reflection in atomic flux lattices, Sci. Adv. 3, e1602685 (2017).

[19] M. Mancini, G. Pagano, G. Cappellini, L. Livi, M. Rider, J. Catani, C. Sias, P. Zoller, M. Inguscio, M. Dalmonte, and L. Fallani, Observation of chiral edge states with neutral fermions in synthetic Hall ribbons, Science 349, 1510 (2015).

[20] E. J. Meier, F. A. An, A. Dauphin, M. Maffei, P. Massignan, T. L. Hughes, and B. Gadway, Observation of the topological
Anderson insulator in disordered atomic wires, Science 362, 929 (2018).

[21] E. J. Meier, F. A. An, and B. Gadway, Observation of the topological soliton state in the Su-Schrieffer-Heeger model, Nat. Commun. 7, 13986 (2016).

[22] F. A. An, E. J. Meier, and B. Gadway, Engineering a FluxDependent Mobility Edge in Disordered Zigzag Chains, Phys. Rev. X 8, 031045 (2018).

[23] E. Lustig, S. Weimann, Y. Plotnik, Y. Lumer, M. A. Bandres, A. Szameit, and M. Segev, Photonic topological insulator in synthetic dimensions, Nature (London) 567, 356 (2019).

[24] D. Jukic and H. Buljan, Four-dimensional photonic lattices and discrete tesseract solitons, Phys. Rev. A 87, 013814 (2013).

[25] X.-W. Luo, X. Zhou, C.-F. Li, J.-S. Xu, G.-C. Guo, and Z.-W. Zhou, Quantum simulation of 2D topological physics in a 1D array of optical cavities, Nat. Commun. 6, 7704 (2015).

[26] L. Yuan, Y. Shi, and S. Fan, Photonic gauge potential in a system with a synthetic frequency dimension, Opt. Lett. 41, 741 (2016).

[27] H. M. Price, T. Ozawa, and H. Schomerus, Synthetic dimensions and topological chiral currents in mesoscopic rings, Phys. Rev. Research 2, 032017 (2020).

[28] S. Barbarino, L. Taddia, D. Rossini, L. Mazza, and R. Fazio, Magnetic crystals and helical liquids in alkaline-earth fermionic gases, Nat. Commun. 6, 8134 (2015).

[29] Z. Yan, S. Wan, and Z. Wang, Topological superfluid and Majorana zero modes in synthetic dimension, Sci. Rep. 5, 15927 (2015).

[30] E. Cornfeld and E. Sela, Chiral currents in one-dimensional fractional quantum Hall states, Phys. Rev. B 92, 115446 (2015).

[31] S. Barbarino, L. Taddia, D. Rossini, L. Mazza, and R. Fazio, Synthetic gauge fields in synthetic dimensions: Interactions and chiral edge modes, New J. Phys. 18, 035010 (2016).

[32] X. Zhang, M. Bishof, S. L. Bromley, C. V. Kraus, M. S. Safronova, P. Zoller, A. M. Rey, and J. Ye, Spectroscopic observation of SU(N)-symmetric interactions in Sr orbital magnetism, Science 345, 1467 (2014).

[33] J. Jünemann, A. Piga, S.-J. Ran, M. Lewenstein, M. Rizzi, and A. Bermudez, Exploring Interacting Topological Insulators with Ultracold Atoms: The Synthetic Creutz-Hubbard Model, Phys. Rev. X 7, 031057 (2017).

[34] T. Bilitewski and N. R. Cooper, Synthetic dimensions in the strong-coupling limit: Supersolids and pair superfluids, Phys. Rev. A 94, 023630 (2016).

[35] S. Greschner and T. Vekua, Vortex-Hole Duality: A Unified Picture of Weak- and Strong-Coupling Regimes of Bosonic Ladders with Flux, Phys. Rev. Lett. 119, 073401 (2017).

[36] J. Xu, Q. Gu, and E. J. Mueller, Realizing the Haldane Phase with Bosons in Optical Lattices, Phys. Rev. Lett. 120, 085301 (2018).

[37] F. A. An, E. J. Meier, J. Ang'ong'a, and B. Gadway, Correlated Dynamics in a Synthetic Lattice of Momentum States, Phys. Rev. Lett. 120, 040407 (2018).

[38] D. Xie, T.-S. Deng, T. Xiao, W. Gou, T. Chen, W. Yi, and B. Yan, Topological Quantum Walks in Momentum Space with a Bose-Einstein Condensate, Phys. Rev. Lett. 124, 050502 (2020).

[39] S. L. Bromley, S. Kolkowitz, T. Bothwell, D. Kedar, A. SafaviNaini, M. L. Wall, C. Salomon, A. M. Rey, and J. Ye, Dynamics 
of interacting fermions under spin-orbit coupling in an optical lattice clock, Nat. Phys. 14, 399 (2018).

[40] M. A. Cazalilla, A. F. Ho, and M. Ueda, Ultracold gases of ytterbium: Ferromagnetism and Mott states in an SU(6) Fermi system, New J. Phys. 11, 103033 (2009).

[41] A. V. Gorshkov, M. Hermele, V. Gurarie, C. Xu, P. S. Julienne, J. Ye, P. Zoller, E. Demler, M. D. Lukin, and A. M. Rey, Twoorbital $\mathrm{SU}(N)$ magnetism with ultracold alkaline-earth atoms, Nat. Phys. 6, 289 (2010).

[42] G. Pagano, M. Mancini, G. Cappellini, P. Lombardi, F. Schäfer, H. Hu, X.-J. Liu, J. Catani, C. Sias, M. Inguscio, and L. Fallani, A one-dimensional liquid of fermions with tunable spin, Nat. Phys. 10, 198 (2014).

[43] D. C. Tsui, H. L. Stormer, and A. C. Gossard, Two-Dimensional Magnetotransport in the Extreme Quantum Limit, Phys. Rev. Lett. 48, 1559 (1982).

[44] R. B. Laughlin, Anomalous Quantum Hall Effect: An Incompressible Quantum Fluid with Fractionally Charged Excitations, Phys. Rev. Lett. 50, 1395 (1983).

[45] R. Prange and S. Girvin, The Quantum Hall Effect (Springer, New York, 1990).

[46] M. Lacki, H. Pichler, A. Sterdyniak, A. Lyras, V. E. Lembessis, O. Al-Dossary, J. C. Budich, and P. Zoller, Quantum Hall physics with cold atoms in cylindrical optical lattices, Phys. Rev. A 93, 013604 (2016).

[47] T. Ozawa and I. Carusotto, Synthetic Dimensions with Magnetic Fields and Local Interactions in Photonic Lattices, Phys. Rev. Lett. 118, 013601 (2017).

[48] C. Chin, R. Grimm, P. Julienne, and E. Tiesinga, Feshbach resonances in ultracold gases, Rev. Mod. Phys. 82, 1225 (2010).

[49] P. Zhang, P. Naidon, and M. Ueda, Independent Control of Scattering Lengths in Multicomponent Quantum Gases, Phys. Rev. Lett. 103, 133202 (2009).

[50] A. M. Kaufman, R. P. Anderson, T. M. Hanna, E. Tiesinga, P. S. Julienne, and D. S. Hall, Radio-frequency dressing of multiple Feshbach resonances, Phys. Rev. A 80, 050701(R) (2009).

[51] For Zeeman sublevels, see Sec. III; spin-changing collisions can be energetically suppressed in the presence of a quadratic Zeeman field.

[52] N. Goldman and J. Dalibard, Periodically Driven Quantum Systems: Effective Hamiltonians and Engineered Gauge Fields, Phys. Rev. X 4, 031027 (2014).

[53] M. Bukov, L. D’Alessio, and A. Polkovnikov, Universal high-frequency behavior of periodically driven systems: From dynamical stabilization to Floquet engineering, Adv. Phys. 64, 139 (2015).

[54] L. W. Clark, A. Gaj, L. Feng, and C. Chin, Collective emission of matter-wave jets from driven Bose-Einstein condensates, Nature (London) 551, 356 (2017).

[55] L. W. Clark, Quantum many-body dynamics with driven Bose condensates: Kibble-Zurek mechanism and Bose fireworks, Ph.D. thesis, The University of Chicago, 2017.

[56] M. Theis, G. Thalhammer, K. Winkler, M. Hellwig, G. Ruff, R. Grimm, and J. H. Denschlag, Tuning the Scattering Length with an Optically Induced Feshbach Resonance, Phys. Rev. Lett. 93, 123001 (2004).

[57] A. J. Moerdijk, B. J. Verhaar, and A. Axelsson, Resonances in ultracold collisions of ${ }^{6} \mathrm{Li},{ }^{7} \mathrm{Li}$, and ${ }^{23} \mathrm{Na}$, Phys. Rev. A 51, 4852 (1995).
[58] D. J. Papoular, G. V. Shlyapnikov, and J. Dalibard, Microwave-induced Fano-Feshbach resonances, Phys. Rev. A 81, 041603(R) (2010).

[59] P. O. Fedichev, Y. Kagan, G. V. Shlyapnikov, and J. T. M. Walraven, Influence of Nearly Resonant Light on the Scattering Length in Low-Temperature Atomic Gases, Phys. Rev. Lett. 77, 2913 (1996).

[60] F. K. Fatemi, K. M. Jones, and P. D. Lett, Observation of Optically Induced Feshbach Resonances in Collisions of Cold Atoms, Phys. Rev. Lett. 85, 4462 (2000).

[61] N. Gross, Z. Shotan, O. Machtey, S. Kokkelmans, and L. Khaykovich, Study of Efimov physics in two nuclear-spin sublevels of ${ }^{7} \mathrm{Li}$, C. R. Phys. 12, 4 (2011).

[62] I. Ferrier-Barbut, M. Delehaye, S. Laurent, A. T. Grier, M. Pierce, B. S. Rem, F. Chevy, and C. Salomon, A mixture of Bose and Fermi superfluids, Science 345, 1035 (2014).

[63] M. P. A. Fisher, G. Grinstein, and D. S. Fisher, Boson localization and the superfluid-insulator transition, Phys. Rev. B 40, 546 (1989).

[64] K. Sheshadri, H. R. Krishnamurthy, R. Pandit, and T. V. Ramakrishnan, Superfluid and insulating phases in an interacting-boson model: Mean-field theory and the RPA, Europhys. Lett. 22, 257 (1993).

[65] D. Jaksch, C. Bruder, J. I. Cirac, C. W. Gardiner, and P. Zoller, Cold Bosonic Atoms in Optical Lattices, Phys. Rev. Lett. 81, 3108 (1998).

[66] M. Greiner, O. Mandel, T. Esslinger, T. Hansch, and I. Bloch, Quantum phase transition from a superfluid to a Mott insulator in a gas of ultracold atoms, Nature (London) 415, 39 (2002).

[67] E. Berg, E. G. Dalla Torre, T. Giamarchi, and E. Altman, Rise and fall of hidden string order of lattice bosons, Phys. Rev. B 77, 245119 (2008).

[68] M. Endres, M. Cheneau, T. Fukuhara, C. Weitenberg, P. Schauß, C. Gross, L. Mazza, M. C. Banuls, L. Pollet, I. Bloch, and S. Kuhr, Observation of correlated particle-hole pairs and string order in low-dimensional Mott insulators, Science 334, 200 (2011).

[69] T. D. Kuhner and H. Monien, Phases of the one-dimensional Bose-Hubbard model, Phys. Rev. B 58, R14741(R) (1998).

[70] T. D. Kühner, S. R. White, and H. Monien, One-dimensional Bose-Hubbard model with nearest-neighbor interaction, Phys. Rev. B 61, 12474 (2000).

[71] A. Läuchli and C. Kollath, Spreading of correlations and entanglement after a quench in the one-dimensional Bose-Hubbard model, J. Stat. Mech.: Theory Exp. (2008) P05018.

[72] M. Heyl, P. Hauke, and P. Zoller, Quantum localization bounds Trotter errors in digital quantum simulation, Sci. Adv. 5, eaau8342 (2019).

[73] S. R. White and A. E. Feiguin, Real-Time Evolution Using the Density Matrix Renormalization Group, Phys. Rev. Lett. 93, 076401 (2004); A. E. Feiguin and S. R. White, Time-step targeting methods for real-time dynamics using the density matrix renormalization group, Phys. Rev. B 72, 020404(R) (2005).

[74] All the t-DMRG results are obtained by fixing the time step $\delta t=10^{-3}\left(1 / J_{\text {eff }}\right)$ and keeping up to 1500 DMRG states in the time evolution. 
[75] M. Atala, M. Aidelsburger, M. Lohse, J. T. Barreiro, B. Paredes, and I. Bloch, Observation of chiral currents with ultracold atoms in bosonic ladders, Nat. Phys. 10, 588 (2014).

[76] S. Greschner, M. Piraud, F. Heidrich-Meisner, I. P. McCulloch, U. Schollwöck, and T. Vekua, Spontaneous Increase of Magnetic Flux and Chiral-Current Reversal in Bosonic Ladders: Swimming against the Tide, Phys. Rev. Lett. 115, 190402 (2015).

[77] M. Piraud, F. Heidrich-Meisner, I. P. McCulloch, S. Greschner, T. Vekua, and U. Schollwöck, Vortex and Meissner phases of strongly interacting bosons on a two-leg ladder, Phys. Rev. B 91, 140406(R) (2015).

[78] E. Orignac, R. Citro, M. Di Dio, S. De Palo, and M.-L. Chiofalo, Incommensurate phases of a bosonic two-leg ladder under a flux, New J. Phys. 18, 055017 (2016).

[79] M. Di Dio, S. De Palo, E. Orignac, R. Citro, and M. L. Chiofalo, Persisting Meissner state and incommensurate phases of hardcore boson ladders in a flux, Phys. Rev. B 92, 060506(R) (2015).

[80] S. Greschner, M. Piraud, F. Heidrich-Meisner, I. P. McCulloch, U. Schollwöck, and T. Vekua, Symmetry-broken states in a system of interacting bosons on a two-leg ladder with a uniform Abelian gauge field, Phys. Rev. A 94, 063628 (2016).

[81] E. J. Bergholtz and Z. Liu, Topological flat band models and Chern insulators, Int. J. Mod. Phys. B 27, 1330017 (2013).

[82] D. Muth, D. Petrosyan, and M. Fleischhauer, Dynamics and evaporation of defects in Mott-insulating clusters of boson pairs, Phys. Rev. A 85, 013615 (2012).
[83] T. Keilmann and J. J. Garcia-Ripoll, Dynamical Creation of Bosonic Cooper-Like Pairs, Phys. Rev. Lett. 100, 110406 (2008).

[84] A. Rosch, D. Rasch, B. Binz, and M. Vojta, Metastable Superfluidity of Repulsive Fermionic Atoms in Optical Lattices, Phys. Rev. Lett. 101, 265301 (2008).

[85] K. Winkler, G. Thalhammer, F. Lang, R. Grimm, J. Hecker Denschlag, A. J. Daley, A. Kantian, H. P. Büchler, and P. Zoller, Repulsively bound atom pairs in an optical lattice, Nature (London) 441, 853 (2006).

[86] N. Strohmaier, D. Greif, R. Jördens, L. Tarruell, H. Moritz, T. Esslinger, R. Sensarma, D. Pekker, E. Altman, and E. Demler, Observation of Elastic Doublon Decay in the Fermi-Hubbard Model, Phys. Rev. Lett. 104, 080401 (2010).

[87] T. Fukuhara, P. Schauss, M. Endres, S. Hild, M. Cheneau, I. Bloch, and C. Gross, Microscopic observation of magnon bound states and their dynamics, Nature (London) 502, 76 (2013).

[88] M. J. Mark, E. Haller, K. Lauber, J. G. Danzl, A. Janisch, H. P. Büchler, A. J. Daley, and H.-C. Nägerl, Preparation and Spectroscopy of a Metastable Mott-Insulator State with Attractive Interactions, Phys. Rev. Lett. 108, 215302 (2012).

[89] P. Barmettler, M. Punk, V. Gritsev, E. Demler, and E. Altman, Relaxation of Antiferromagnetic Order in Spin-1/2 Chains Following a Quantum Quench, Phys. Rev. Lett. 102, 130603 (2009).

[90] K. M. Jones, E. Tiesinga, P. D. Lett, and P. S. Julienne, Ultracold photoassociation spectroscopy: Long-range molecules and atomic scattering, Rev. Mod. Phys. 78, 483 (2006). 